\title{
Participatory Design for Cultural Representation: A Cultural Transparency Perspective
}

\author{
Amalia G. Sabiescu ${ }^{1}$ and Nemanja Memarovic ${ }^{2}$ \\ ${ }^{1}$ New Media in Education Laboratory, Faculty of Communication Sciences, \\ Università della Svizzera italiana, Via G. Buffi 13, 6900 Lugano, Switzerland \\ ${ }^{2}$ Faculty of Informatics, Università della Svizzera italiana, Via G. Buffi 13, \\ 6900 Lugano, Switzerland \\ \{sabiesca, nemanja.memarovic\} @usi.ch
}

\begin{abstract}
Participatory design approaches are being increasingly employed for designing digital artefacts and information systems with and for local communities. These cases require a reconceptualization of PD processes to account for widened knowledge gaps between designers and community members, and new patterns of community-defined design goals. In this paper we provide a perspective on the design process that will help designers to better plan their involvement in participatory projects with local communities. Our analytical stance resides on an interpretation of Étienne Wenger's theory of cultural transparency. Participatory design is analysed as an iterative process of decoding and encoding that involves users/local people and designers having as outcome understanding (through decoding) and representations (through encoding). Cultural transparency, achieved when the two agents advanced sufficient understanding on the other's practices, is the landmark for effective design. The paper argues for the importance of working towards attainment of cultural transparency in community-based projects, in particular when the goal is to create culturally representative artefacts. Examples of activities and suggestions for advancing cultural transparency in these contexts are provided.
\end{abstract}

Keywords: participatory design, cultural transparency, cross-cultural design, local communities, cultural representation.

\section{Introduction}

Participatory design (PD) approaches, traditionally used in work environments [1], are being increasingly employed for designing digital artefacts with and for local communities, serving goals defined by community interests, needs, and aspirations. For example, ICTs can be instrumental for preservation and transmission of local knowledge among different generations of the same community [2, 3], recovery of memory by appropriation and digital representation of community artefacts [4], and networking among disenfranchised indigenous communities [5]. These goals can be supported by the design and development of various information spaces, communication artefacts, and repositories, ranging from digital archives to community websites. When using PD in community contexts, three inter-related sets of challenges arise: 
1. The knowledge gap between designers and communities is widened. For designers, community settings are characterized by peculiar social and cultural features that need to be understood for creating relevant artefacts [3]. For community members, digital technology and design workflows, processes and terminology are replete with unknowns that make their informed participation difficult.

2. A reconceptualization and renegotiation of established design methods and techniques is required for accommodating the participation of community members that may lack digital literacy or even be illiterate. As Winschiers [6] argues, even widely accepted PD methods, such as Future Workshop, may be unfit in certain community contexts.

3. The formulation of design goals needs to take into account relations between the features of the digital artefact and the community's long-term goals and bounding cultural protocols. For example, Christen [4] reports on the design of an indigenous archive in which different levels of access were defined reflecting restricted access to local knowledge for different community groups.

This paper outlines a theoretical perspective that can shed light on the factors that are brought to bear when employing PD in community contexts. Drawing on an interpretation of Étienne Wenger's theory of cultural transparency [7,8], the paper will provide a conceptual mapping of the design continuum highlighting the dynamics of interaction between users and designers in the creation of novel artefacts. PD is analysed as an iterative process of decoding and encoding involving users and designers having as outcome understanding (through decoding) and representations (through encoding). Cultural transparency, achieved when the two agents advanced sufficient understanding on the other's practices, is the landmark for effective design. The paper argues for the importance of working towards attainment of cultural transparency in community contexts, in particular when the goal is to create representative artefacts.

\section{Background and Related Work}

The outline of the theoretical perspective exposed herein was shaped by considerations around a participatory project that aimed to explore the potential of ICTs for giving voice to minority groups starting from the grassroots [9,10]. The project, titled Romani Voices involved two Romani communities in rural Romania. The methodological approach employed placed an emphasis on emergence and people participation in design at two levels: (1) Activity design: people's views were integrated in the design of a content production experience for documenting local cultural practices and collective priorities; and (2) Product design: people participated in designing the information architecture and deciding upon suitable content to be included in a community website for each group involved (www.romanivoices.com).

The study investigated the methodological implications of design interventions in community contexts, and assessed in particular: (1) the conditions, patterns, and impacts of community participation in design; and (2) the challenges of designing culturally representative digital artefacts. Drawing on Manovich [11], culturally representative refers to the quality of a digital artefact or system to properly reflect local 
ways of knowing and being, and construct in the mind of the onlooker the image of a referent existing in reality, in this case a community's socio-cultural and historical features. In an expanded sense, culturally representative also stands for the capacity of a system or artefact to advance locally defined goals for information, expression and communication.

To investigate these aspects, we surveyed the more generic PD literature, as well as the narrower body of research focusing on community-based participatory design. The PD literature contains a number of contributions that conceptualize the design process around the designers' and the users' inputs. Muller [1] describes PD as the third space in HCI, a space in between the developers' and the users' worlds. He makes an overview of "hybrid practices", PD techniques and methods that fall in between the users' and the developers' world. Methods such as workshop, drama, storytelling, and design games contribute to challenging assumptions and driving novel ideas emerged through dialogue and negotiation among users and developers. Kensing and Munk-Madsen's [12] model of user-developer communication in PD depicts design as a bridge across two different worlds: the users' (the workplace) and the developers' (the technological options). A third domain - the new system - is created throughout the project.

Community-based design studies are characterized, furthermore, by an increased sensitivity to contextual conditions. It has been argued that designing for local communities involves working with and accounting for two different and at times incompatible ways of knowing [2,3]. When the aim is to create culturally representative and locally useful artefacts, design relies on embracing a local viewpoint and allowing activities and design solutions to emerge from it [2]. Community-based design studies, in particular if conducted in indigenous contexts, agree that community participation is the real measure in the development of community-representative artefacts $[2,3,5]$. Community-centric design is not about applying the right method, it rather implies re-interpreting all articulations of the design process in the light of the specific situation [3,13]. Design in community contexts calls for the creation of a new space where design approaches and techniques are re-configured during the interaction between local members and the design team [13].

These considerations frame the position embraced in this paper, and further call for the central questions it poses: if participatory design in community contexts is an emanation of present interactions between designers and local people, can we identify the most important factors underpinning this interaction? In particular, what are the pre-conditions for effective joint work of designers and local communities in the creation of novel artefacts? To answer these questions, the next section introduces an analytical model of the design continuum drawing on Wenger's theory of cultural transparency [7,8], illustrated by vignettes from the Romani Voices study.

The term community is used in the forthcoming part with two different meanings: (1) the term "community" on its own or in the constructs "designers' community" and "users' community" reflects the meaning invested by Wenger [7,8], and indicates a group of people with common interests engaging in shared practices; (2) "Local community" is defined as a construct blending two features: shared geographical 
space, and members' relatedness [14], therefore referring to groups of people living in the same space and engaging in social interaction and shared activities.

\section{The Theory of Cultural Transparency and PD}

Étienne Wenger's theory of cultural transparency [7,8] presents interest for conceptualising PD due to the perspective it provides on the interplay between learning, participation and meaning production. The theory can be summed-up in five core statements: (1) Knowledge is not existing in the abstract but is constantly produced and enacted as part of the practice of communities, taken to be broadly groups of people that engage in shared activities around a profession or common interest; (2) The practices of a community result in the production of objects/representations invested with meaning (from symbols, terms and procedures to gestures and tangible artefacts); (3) Representations are the results of processes of reduction, simplification or meaning investment, meant to facilitate members' joint activities and interaction. They are imbued with meaning, but are at the same time hermetic for a new-comer; (4) Understanding the meaning of representations, or learning, can only happen through direct participation in the practices of a community; (5) Cultural transparency refers to the quality that objects stemming from the practice of communities acquire when their cultural significance is unveiled by an agent. It indicates the successful outcome of a process of learning, on virtue of which a community member can handle representations in a seamless manner. The term "cultural" is used to convey the context-specific and perspective-bound character of knowledge and learning [7, p. 104].

The sections below (1) outline an interpretation and operationalization of Wenger's cultural transparency theory through encoding and decoding meaning, (2) show its relevance for design, specifically $\mathrm{PD}$, and (3) suggest implications for conducting effective PD processes in community contexts.

\subsection{Encoding and Decoding Meaning}

Central to Wenger's theory are two alternate processes that characterize learning through participation in a community of practice: (1) members' regular engagement in activities or practices relying on the production and use of representations; and (2) the new comer's attempt to learn and seize the meanings embedded in these representations. We can look at these alternate processes of creation vs. understanding of representations as a process of encoding vs. decoding meaning and significance. (Note: The encoding-decoding interpretation is part of the authors' approach and was not used by Wenger.)

1. Encoding: Objects and representations are created through abstraction and simplification of the practices in a given community. In this process, they are invested with significance that is invisible to an outsider's eye.

2. Decoding: The attempt to understand the significance of objects or representations of a community equals to a process of decoding, where an outsider tries to make out the hidden layers of meaning in a representation. This understanding is 
possible, in Wenger's view, only through participation in the practices of a community, and is entirely achieved in full participation. Decoding activities build up understandings until reaching cultural transparency.

\subsection{The Perspective on Design}

Design aims to produce useful artefacts for a certain class or community of users, usefulness which can be determined only in direct relation to the user activities that the artefact will support. Effective design needs to build on a thorough understanding of the user activities that its outcome will serve. In PD, this understanding is advanced by enabling users' direct participation in design activities.

Based on the theory of cultural transparency, participatory design brings together the members of two communities: designers and users, each with a history of past intra-community practices and handling representations created on virtue of such practices. In instantiating PD, a new space of interaction is created, in which the practices and representations of each group are at the outset little known for the other (Fig. 1). PD activities alternate constantly between decoding activities, having as outcome understandings of the other community, and encoding activities, having as outcome new design representations. Decoding activities serve to build up to increased understanding until cultural transparency is reached, indicating the point where each community understands each other's representations sufficiently for engaging in fruitful design activities. The main thesis put forward in this paper is that effective design starts when cultural transparency is reached. The underpinnings and implications of this position are outlined below.

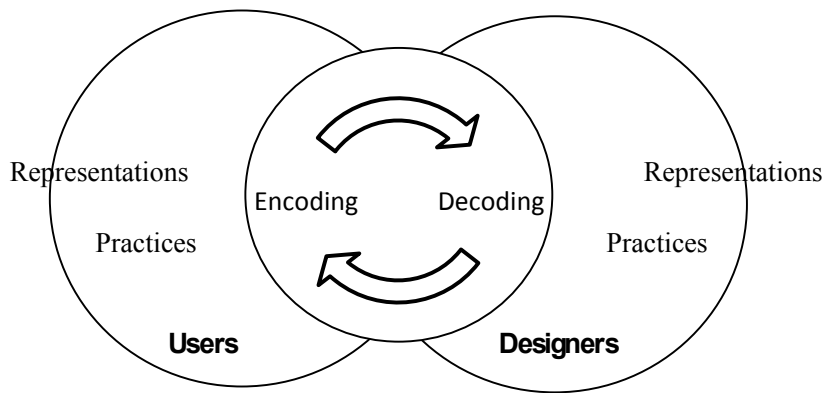

Fig. 1. Participatory design as an iterative process of encoding and decoding involving users and designers

Decoding and Encoding in the PD Process. Decoding activities can be categorized with respect to two dimensions: the agent, or who engages in learning (users or designers); and in relation to which area of practice (users' or designers' community). There result four types of activities: (1) Users understanding design (e.g. through demos, informative sessions); (2) Users deriving new understandings of their own context (e.g. through active questioning and reflective sessions); (3) Designers understanding the local context (e.g. through ethnographic methods); (4) Designers 
deriving new understandings of design in the light of the local context (e.g. through discussions on contextual applications of design solutions).

Encoding activities serve to create new representations as part of the design process. While Wenger uses the terms "objects" and "representations" interchangeably to encompass all classes of objects resulting from practice, we propose that for being useful in analysing design processes, two distinctions are necessary:

1. Based on level of concreteness, representations can be abstract (e.g. design terminology, work procedures) or concrete (e.g. written codes, manuals).

2. Based on role in the PD process, there can be mediating and final representations. Mediating representations act as intermediary in the relationship between agents (such as a procedure telling agents what each should do) or in the activity performed by agents (as in the use of a mock-up for advancing understanding of the functionalities of the final system). Final representations are those that users will actually be utilizing, such as the final system (Table 1).

Table 1. Examples of types of representations produced during PD, according to level of abstractness (abstract - concrete) and role in the PD process (mediating - final)

\begin{tabular}{|l|l|l|}
\hline & Mediating & Final \\
\hline Abstract & Design terms, work procedures & Workflows based on product usage \\
\hline Concrete & Mock-up & Final system \\
\hline
\end{tabular}

Mixed activities, blending decoding and encoding can be employed in order to generate experiential knowledge and showcase or probe preliminary outcomes of design activities. Examples are cultural probes [15], in which users employ low or digital technology to document their context and produce representations that can trigger group discussions and reflections. In Romani Voices, local people used video technology to record snapshots of their life and traditions that were therefore discussed with the field researcher. This served a three fold purpose: (1) local people generated new understandings of their context; (2) the field researcher derived understanding of the local context through people's perspectives; and (3) local people got acquainted with the use of digital technology. Another example is the creation of a community blog and the website mock-up, both of which served to enhance understanding of design-specific terms and artefacts for local people.

A further delineation of activity types can be done by specifying the dynamics of interaction between users and designers in performing either decoding or encoding activities. These activities can be performed separately by each agent (e.g. a designer learning about the local context through non-participant observation) or jointly (e.g. the deployment and joint assessment of an information system). The latter can be referred to by Wenger's concept of boundary practice, indicating the sphere of interaction created through the involvement of users and designers in common activities. A boundary practice is at the same time (1) a fertile field for enhancing understanding of representations commonly grounded in the domain of each community; and (2) the field of production of new meanings. Just as any practice of a community, it can generate its own representations, understood jointly by designers and users. PD activities 
performed by users, designers, or jointly move constantly between the distinct areas of practice of the two communities and the boundary practice area.

The Limits of Participation. The forms that participation in design can take can be related to Wenger's concept of legitimate peripheral participation - LPP [7,8]. LPP as an analytical category refers to learning through increased participation in the practices of a community. However, an agent may participate in practices without attempting to become a member. Her/his position is clearly outlined and so are the limits to her/his participation, based on agreed protocols with the users. In PD, the limits of participation are defined in relation to the degree of knowledge needed by users and designers in order to cooperate effectively in the achievement of the design goals.

\subsection{Implications for Design with Local Communities}

The main argument put forward in this paper is that cultural transparency is the landmark for effective design. In PD, cultural transparency indicates the outcome of a process of learning focused on acquiring knowledge circumscribed to two spheres the users' context and ICT design. Users will be able to take informed decisions regarding the new system only after having seized the meanings and significance of design representations (terminology, procedures, rules, artefacts). Designers, on the other hand, will be able to put forward adequate solutions when they have reached an understanding of users' practices.

Cultural transparency as landmark for effective design becomes critical when PD is employed in community contexts, aiming to produce artefacts that can advance longterm community goals. When design artefacts are intended to serve cultural representation as defined earlier, there is a need to draw on a common pool of understandings enabling relations between community practices and design solutions. The analytical model introduced drives attention to three aspects to be considered:

1. The types of activities employed in $P D$, which may fall in three categories: decoding, encoding, and mixed. We argue in particular for the usefulness of mixed activities (e.g. cultural probes), in which mediating representations are created for advancing experiential knowledge and understanding the applicability of design solutions to a specific context.

2. The sequence of decoding, encoding, and mixed activities. Effective sequencing requires sensitivity and attention to the type of knowledge and understandings required for taking informed decisions in design. We suggest that in the beginning of a project cyclic iterations of decoding and encoding activities concerned with the production of mediating representations are effective drivers of understanding on both sides. On this basis, encoding-intensive activities may further be devised.

3. The value of boundary practices. When engaging in joint activities, in particular those blending decoding and encoding (e.g. engaging in the deployment and discussion of cultural probes), users and designers instantiate shared spaces of interaction, with their own production of meaning. These spaces are particularly fruitful for advancing understandings of design, the local context, and design solutions grounded in the needs of the local context. 


\section{Conclusion}

This paper introduced an analytical model for PD processes, drawing on Wenger's theory of cultural transparency. The model depicts the PD continuum as an alternation of activities in which both users and designers engage, respectively for (1) deriving knowledge and understanding of the other's area of practice and (2) investing meaning in the creation of new representations. The model is proposed as an analytical tool for understanding the factors that are brought to bear and conditions to be taken into account when engaging in PD projects in new contexts, for instance with local communities. The importance of reaching a shared sphere of understandings, indicated by the term cultural transparency, is especially critical when design interventions are aimed to produce culturally representative artefacts that will help to advance a community's goals and can be integrated and used in its practices.

\section{References}

1. Muller, M.J.: Participatory design: The third space in HCI. In: The Human Computer Interaction Handbook: Fundamentals, Evolving Technologies and Emerging Applications, pp. 1051-1068 (2002)

2. Rodil, K., Winschiers-Theophilus, H., Jensen, K.L.: Enhancing cross- cultural participation through creative visual exploration. In: Procs. PDC 2012, pp. 81-90. ACM (2012)

3. Verran, H., Christie, M., Anbins-King, B., van Weeren, T., Yunupingu, W.: Designing Digital Knowledge Management Tools with Aboriginal Australians. Performative Knowledge Making. Charles Darwin University, Australia (2006)

4. Christen, K.: Archival Challenges and Digital Solutions in Aboriginal Australia. The SAAA Archeological Record. Special Issue International Cooperative Research 8(2), 2124 (2008)

5. Srinivasan, R.: Where Information Society and Community Voice Intersect. The Information Society 22(5) (2005)

6. Winschiers, H.: The challenges of participatory design in an intercultural context: Designing for Usability in Namibia. In: Proc. PDC 2006 (2006)

7. Wenger, E.: Towards a Theory of Cultural Transparency. Unpublished Doctoral Dissertation. Department of Sociology. University of California, Irvine (1991)

8. Wenger, E.: Communities of Practice: Learning, Meaning, and Identity. Cambridge University Press, Cambridge (1998)

9. Sabiescu, A.G.: Exploiting the Intergenerational Connection in Community Media Initiatives for Minority Cultures: A Case Study. International Journal of Technology, Knowledge and Society 7(6), 1-18 (2012)

10. Sabiescu, A.G.: The Issue of Collective Agency in Community-based Open Content Creation. In: Proc. IDIA 2011 (2011)

11. Manovich, L.: The language of new media. MIT Press, Cambridge (2001)

12. Kensing, F., Munk-Madsen, A.: PD: Structure in the toolbox. Com. of the ACM 36(6), 7885 (1993)

13. Van der Velden, M.: Design for the contact zone: Knowledge management software and the structure of indigenous knowledges. In: Proc. CATaC 2010, pp. 1-18 (2010)

14. Gusfield, J.R.: The community: A critical response. Harper Colophone, New York (1975)

15. Gaver, B., Dunne, T., Pacenti, E.: Cultural Probes. Interactions 6(1), 21-29 (1999) 